\title{
A reduced thermodynamic description of phase separation in a quasi-one-dimensional granular gas.
}

\author{
James P. D. Clewett, R. M. Bowley, and Michael R. Swift \\ School of Physics and Astronomy, University of Nottingham, NG7 2RD, UK
}

\begin{abstract}
We describe simulations of a quasi-one-dimensional, vibrated granular gas which exhibits an apparent phase separation into a liquid-like phase and a gas-like phase. In thermal equilibrium, such a phase separation in one-dimension is prohibited by entropic considerations. We propose that the granular gas minimises a function of the conserved mechanical variables alone: the particle number and volume. Simulations in small cells can be used to extract the equation of state and predict the coexisting pressure and densities, as confirmation of the minimisation principle. Fluctuations in the system manifest themselves as persistent density waves but they do not destroy the phase-separated state.
\end{abstract}

Systems driven far from equilibrium often exhibit complex collective behaviour such as phase-separation, pattern formation and self-organisation [1-3]. In thermodynamic equilibrium, systems evolve to minimise the appropriate free energy, a principle that lies at the heart of statistical mechanics [4]. No such principle can be generally applied to explain emergent behaviour in farfrom-equilibrium systems.

One of the simplest possible realisations of a far-fromequilibrium system is that of a driven gas of identical spheres that undergo dissipative collisions, namely a granular gas $[5,6]$. In both experiment and simulation, non-ideal behaviour analogous to a liquid-gas phase separation with an emergent surface tension has been observed in a confined, periodically vibrated, quasi-2D system $[7,8]$. An equal-areas construction was employed to predict the coexisting densities to a reasonable degree of accuracy [9]. However, the validity of this approach has been called into question due to a breach of detailed balance [10]. Similarly, the breakdown of detailed balance in the interfacial region has been argued to explain an unequal-areas rule in active Brownian particles [11].

A quasi-1D granular gas driven by thermalised boundaries also exhibits a phase separation analogous to a van der Waals gas in equilibrium [12-15]. The general applicability of an equal-areas rule in this case has been questioned due to the proximity of the critical point [16]. In thermodynamic equilibrium a 1D gas of particles with short-ranged interactions would not be expected to phase separate at all, due to the dominance of thermal fluctuations. As such the existence of a 1D phase separation in granular matter illustrates a striking difference between equilibrium and non-equilibrium systems. Can a modified version of thermodynamics provide a framework to describe phase separation in a 1D granular gas?

In this article we describe simulations of a confined, periodically-vibrated, quasi-1D granular gas, carried out to investigate phase separation in a one-dimensional nonequilibrium system. Surprisingly, we find that this system can be described by a reduced form of thermodynamics in which kinetic, thermal, variables play no significant role.

Our main results are as follows. (i) A finite-size scaling analysis shows that the system exhibits a true $1 \mathrm{D}$ phase separation into a liquid-like phase and a gas-like phase. (ii) We propose that this two-phase coexistence can be described by a function of state $W(N, V)$, where $N$ is the number of particles in the cell and $V$ is the volume of the cell; in the steady state $W(N, V)$ is minimised. (iii) Simulations in small cells can be used to extract the pressure as a function of the volume per particle, $p(v)$, which acts as a non-isothermal equation of state. For a range of small system sizes, an equal-areas construction on $p(v)$ can be used to determine the coexisting pressure and densities, justifying the above assumption. (iv) Gradient terms may be added to $W(N, V)$ to construct an energy functional which accurately describes the steady-state, interfacial profile. (v) In this non-equilibrium steady state a single domain of the dense phase persists and exhibits periodic oscillations in its length with a dominant frequency.

We have carried out computer simulations of $N$ spheres of diameter $d$ in a cell of height $10 d$, length $L$ in the range $10 d$ to $25600 d$ and thickness $d$ (to confine the motion of the spheres to a plane)[17]. The cell is shaken sinusoidally in the direction of the height with an amplitude, A, typically of around $1.2 d$. The frequency of vibration is $50 \mathrm{~Hz}$, the diameter $d=1 \mathrm{~mm}$ and the particle density was taken to be that of bronze. Periodic boundary conditions are imposed in the long direction. The effects of gravity and friction are ignored. Particle interactions are modelled by a linear spring-dashpot force, with the coefficient of restitution equal to $\varepsilon=0.7$. Our findings are robust to changes in $\varepsilon$, the addition of gravity, the geometry of the cell and the mass of the particles.

In thermodynamic equilibrium, it is often difficult to determine the nature of a phase transition due to finite size and time effects [18]. In the following paragraphs we describe a number of tests that we have carried out in order to demonstrate that our system exhibits a true phase separation.

Initially, the particles are positioned randomly within the cell and given a small random velocity. The cell is shaken. For a range of amplitudes and mean number densities the particles spontaneously phase separate in the direction perpendicular to the vibration, $x$ [19]. This 

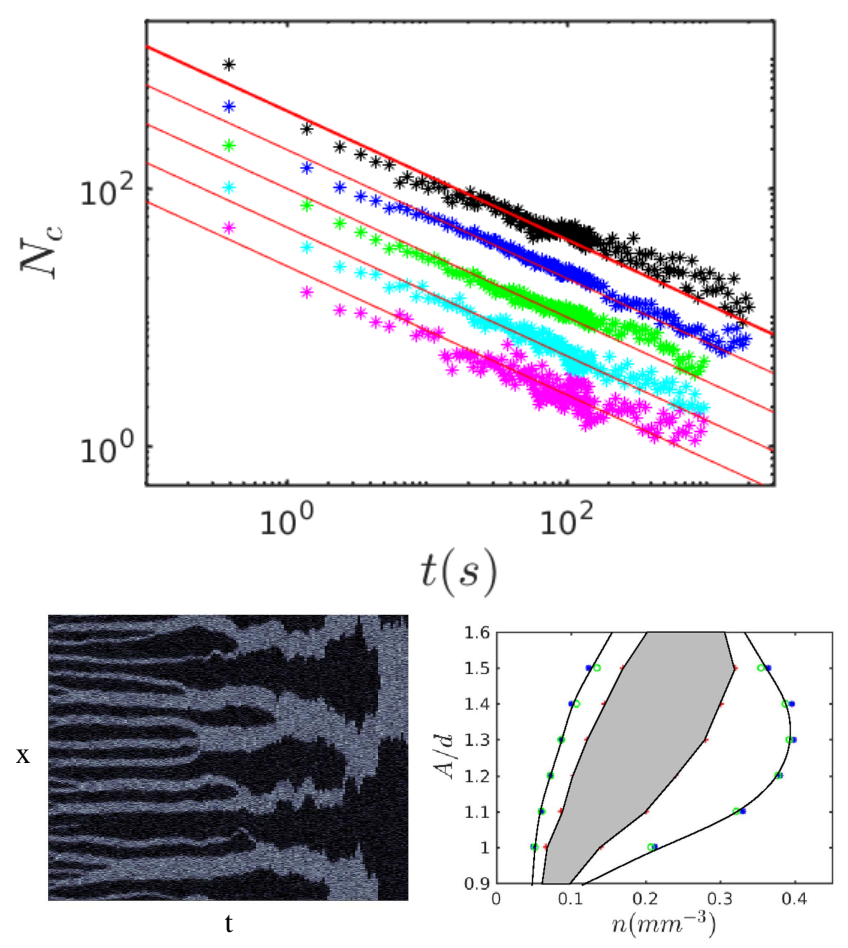

FIG. 1. The top panel shows the variation of the number of independent clusters with time for systems of lengths $L / d=1600,3200,6400,12800,25600$ (from bottom to top). The lines have slopes of $-1 / 2$. The lower left panel shows a space-time plot of the density to illustrate the coarsening dynamics (the dense phase is shown by the lighter shading). The lower right panel shows the phase diagram in the space of $A / d$ and number density $n$. The shaded area indicates the spinodal region and the outer lines correspond to the binodal densities.

behaviour is shown in the space-time plot of the local number density given in the lower left panel of Fig. 1. The main panel of Fig. 1 shows the number of independent clusters, $N_{c}$, as a function of time, $t$, for different length systems with mean particle number density $0.1875 \mathrm{~mm}^{-3}$. The lines all have a constant slope, showing that $N_{c}$ varies as $t^{-1 / 2}$. The corresponding mean cluster size thus grows as $t^{1 / 2}$, in contrast to the observed behaviour in 2D, where for the Cahn-Hilliard model with a conserved order parameter we would expect the length scale to grow as $t^{1 / 3}[20]$.

By considering the behaviour for a range of system sizes, we observe that there is no evidence of a particular length-scale appearing in the system over the times accessible in simulation, of the order 1000 seconds. In the limit of an infinitely long system it is reasonable to infer that the system will coarsen into a single, dense domain within a dilute gas of particles. We also observe that the number of clusters at a given time scales linearly with the length of the system, indicating the extensivity of the phases that form.

The lower right panel in Fig. 1 shows the phase di- agram as a function of amplitude $A$ (expressed as $A / d$ ) against the mean number density, $n$, obtained from direct simulation and from the equation of state discussed below. The shaded area indicates the spinodal region where the system becomes unstable, and the binodal lines show the densities it goes to in the phase-separated state. For a given amplitude, the coexisting densities are independent of the mean number density in the cell. Furthermore, the pressure, calculated using the virial expression [21], is uniform across the cell to a good approximation, having only small fluctuations in space and time. It is also independent of the mean number density.

In thermodynamic equilibrium, a non-ideal gas with short-ranged interactions does not exhibit a phase transition in one dimension. In contrast, our tests have demonstrated that the dissipative granular gas can exhibit a true phase separation. However, the granular temperature i.e. the mean kinetic energy per particle is different in the two phases. Consequently, there is no need to introduce the concept of a granular entropy as the total kinetic energy is not conserved. Hence we propose that our system may be described in terms of a reduced form of thermodynamics based on conserved variables alone.

Consider a box shaken at a constant amplitude, $A$, containing particles in a state in which it has phase separated into a dilute and a dense phase [22]. The dilute phase has a number density $n_{1}=N_{1} / V_{1}$ and the dense phase has density $n_{2}=N_{2} / V_{2}$. Particles can move from one phase to the other, resulting in fluctuations in the particle numbers and volumes of each phase.

For our granular system the total number of particles, $N$, and the total volume, $V$, are fixed. We postulate that there exists an extensive function of state of these conserved variables $W(N, V)$ which, in the steady state, is minimised for a given amplitude of vibration. Note that the total kinetic energy of the particles, $E$, is not fixed so that $W(N, V)$ is not explicitly a function of $E$. For the whole system the total $W$ is

$$
W_{T}=W\left(N_{1}, V_{1}\right)+W\left(N_{2}, V_{2}\right),
$$

with $N=N_{1}+N_{2}$ and $V=V_{1}+V_{2}$.

Minimising $W_{T}$ by varying $N_{1}$ and $V_{1}$ subject to the constraints of fixed particle number and fixed volume leads to

$$
\begin{aligned}
& \left.\frac{\partial W}{\partial N}\right|_{1}=\left.\frac{\partial W}{\partial N}\right|_{2}=\mu^{*}, \\
& \left.\frac{\partial W}{\partial V}\right|_{1}=\left.\frac{\partial W}{\partial V}\right|_{2}=-p^{*},
\end{aligned}
$$

where we define $\mu^{*}$ and $p^{*}$ to be, respectively, the granular chemical potential and granular pressure at two-phase coexistence. Away from coexistence we assert that

$$
p=-\frac{\partial W}{\partial V} \quad \text { and } \quad \mu=\frac{\partial W}{\partial N},
$$

so that $\mathrm{d} W=-p \mathrm{~d} V+\mu \mathrm{d} N$. Extensivity of $W$ requires $W=-p V+\mu N$. Hence, by introducing the intensive 
variables $w=W / V$ and $n=N / V$, these equations become

$$
n \frac{d w}{d n}-w=p
$$

which can be solved for $w(n)$ for a given pressure curve $p(v)$, where $v=1 / n$ is the volume per particle.

To proceed further it is necessary to determine the equation of state, $p(v)$. We use a small-box analysis to measure $p(v)$ for systems shaken at fixed amplitude $A$, the appropriate external constraint imposed on this system. By using sufficiently small boxes with periodic boundary conditions, phase separation can be suppressed, and $p(v)$ can be determined for all values of $v$, not only those on the stable branches. A similar approach was employed by Hansen and Verlet for an equilibrium Lennard-Jones fluid in a restricted fluctuation ensemble [23]. In our granular gas interactions are short ranged, so the use of small boxes to restrict fluctuations is expected to provide an accurate estimate of $p(v)$ [24].

Figure 2 shows $p(v)$ for different length systems ranging from $L=10 d$ to $L=100 d$, shaken with $A=1.2 d$, an amplitude well away from the critical points. The curves exhibit a mechanically unstable region similar to the well-known van der Waals loop in equilibrium. For longer systems the 'shoulder' in the spinodal region arises due to incomplete phase-separation (green); as the system reduces in length the shoulder goes away. For the smallest systems considered (blue) the densities in the bulk phases become system-size dependent due to finitesize effects.

Given the non-monatonic form of $p(v), w(n)$ is a nonconvex function of $n$. If the system acts to minimise $W(N, V)$, a double-tangent construction can be used to determine both $p^{*}$ and $\mu^{*}$, and the coexisting number densities $n_{1}$ and $n_{2}$. The equality of $\mu$ in the two phases implies that the coexisting pressure obeys an equal-areas construction on the equation of state $p(v)$. We emphasise again that the granular temperature depends on the number density; hence $p(v)$ does not correspond to an 'isotherm' as it would for an equilibrium system.

The inset shows the fractional difference in the pressure obtained from an equal-areas construction, $p^{*}$, and the measured pressure, $p_{L}$, for a long system which exhibits complete phase separation. Over the range of system-size considered the deviations from the equal-areas construction, $\Delta p=\left(p^{*}-p_{L}\right) / p_{L}$, is zero to an accuracy of better than $\pm 1.2 \%$. Moreover, if we ignore curves strongly influenced by either phase-separation or finite-size effects, as carried out in equilibrium simulations [23], the value of $\Delta p$ for the black curves is accurate to within less than $0.3 \%$.

Figure 1 shows the binodal curves obtained from smallbox simulations and the equal-areas construction (green open circles), and those obtained directly from the densities in a phase-separated system (blue filled circles). The agreement is very good throughout the phase diagram. These findings show that the predictions based solely on

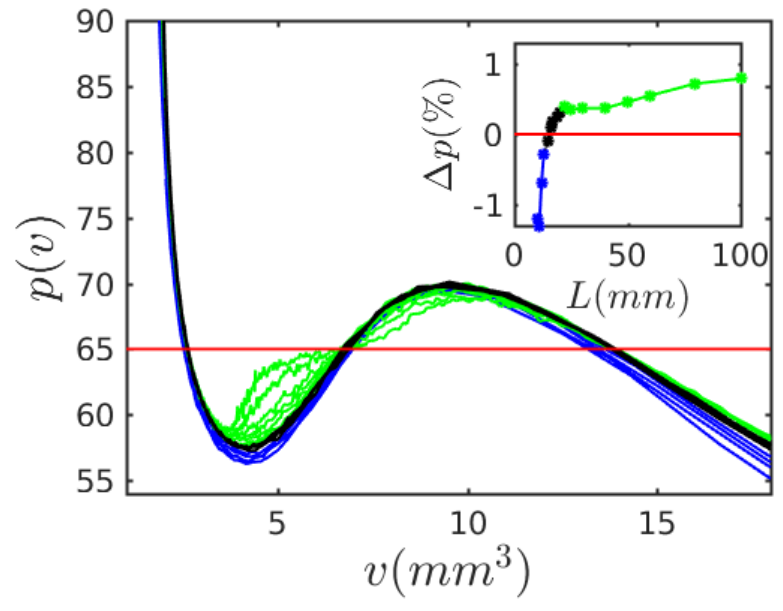

FIG. 2. The main panel shows pressure characteristics $p(v)$ in Pascals obtained from simulations in small boxes, for lengths ranging from $100 d$ down to $10 d$. For larger boxes (green) the curves deviate in the spinodal region due to partial phase separation. For smaller boxes (blue) the curves deviate in the bulk phases due to finite-size effects. The inset shows the percentage deviation in the pressure measured in a phase separated system and that obtained assuming an equal-areas construction on $p(v)$ is valid, as a function of the small box length.

minimising $W(N, V)$ appear to hold for this system to a very high degree of precision, even when the system is well away from the critical point.

An explicit expression for $w(n)$ can be obtained by direct integration of eqn (5). The resulting excess mechanical work $\Delta w(n)=w(n)-\mu^{*} n+p^{*}$ has two equal minima $\Delta w\left(n_{1}\right)$ and $\Delta w\left(n_{2}\right)$ at zero as obtained from the double tangent or equal-areas construction [24]. Physically, minimising $\Delta w$ corresponds to minimising any mechanical work that could be released in exchanging particles from one phase to another at the coexistence pressure $p^{*}$. As the system is dissipative, it is natural to expect that in the absence of fluctuations the system would relax to this minimum energy state. Note that the same equal-areas construction applies in thermodynamic equilibrium, but only if $p(v)$ is an isotherm. In this case the mechanical work function defined here is equivalent to the Helmholtz free energy.

Figure 3 shows $\Delta w(n)$ as a function of number density obtained from $p(v)$ for a small cell, $L=16 d$ and $A / d=$ 1.2. The inset to Fig. 3 shows the time-averaged density profile in the corresponding system in a long cell that allows phase separation (green points). The shape of the interface was measured relative to the point where the local density is mid-way between the bulk liquid and gas densities, thereby negating the effects of interface motion. Many instantaneous configurations were then averaged to give the mean profile.

In equilibrium thermodynamics the interfacial profile can be constructed directly from the excess free energy 


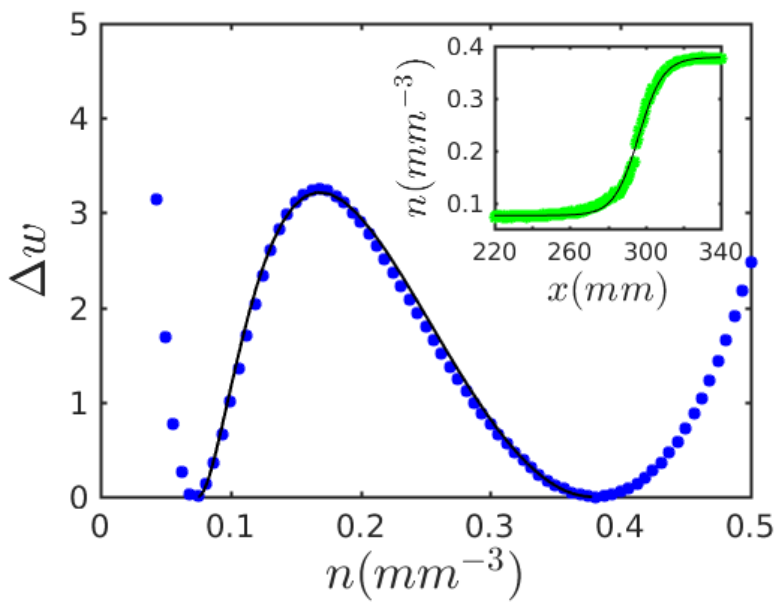

FIG. 3. The main panel shows the excess residual mechanical work, $\Delta w$, in Pascals obtained from $p(v)$ in a small cell (points). The inset shows the mean density profile in a phaseseparated system (green points) and a fit to the profile (black line). The solid line in the main panel shows that minimisation of the mechanical work functional can be used to construct the interfacial profile, as described in the text.

density, and the assumption of a gradient squared term in the free energy functional [24]. If our granular system can be treated in the same fashion, we postulate the existence of a mechanical work functional of the form

$$
W[n]=\int_{-\infty}^{\infty}\left\{\frac{k(n)}{2}\left(\frac{d n}{d x}\right)^{2}+\Delta w(n)\right\} d x,
$$

in terms of the density profile $n(x)$. Minimisation of $W[n]$ with respect to density variations give the EulerLagrange equation satisfied by the interfacial profile [24]. The first integral of this equation, combined with the gradient squared term obtained from the interfacial profile measured in simulations, allows us to estimate $k(n)$. The solid line in the main panel of Fig. 3 shows this fit where we have taken $k(n)$ to be of the form $\kappa / n^{2}$. The agreement is extremely good, given that there are only two adjustable parameters, $\kappa$ and the exponent which we find to be 2. Similar agreement is found for other values of the driving amplitude, provided the system is sufficiently far from the critical regions.

In our granular system a local energy functional is sufficient to provide a macroscopic description. However, an energy functional constructed solely from the pressure has no obvious connection to any large-deviation functional [25] and, as such, does not directly provide information about the fluctuations in the system. In order to investigate the fluctuations in the steady state we consider a system initialised in the phase-separated state and vibrated with $A=1.2 d$. Such a configuration remains as a single dense domain, but the length of the domain exhibits breathing-like modes of oscillation [19].

To characterise these persistent fluctuations we have taken time-series of the domain length of the dense phase,

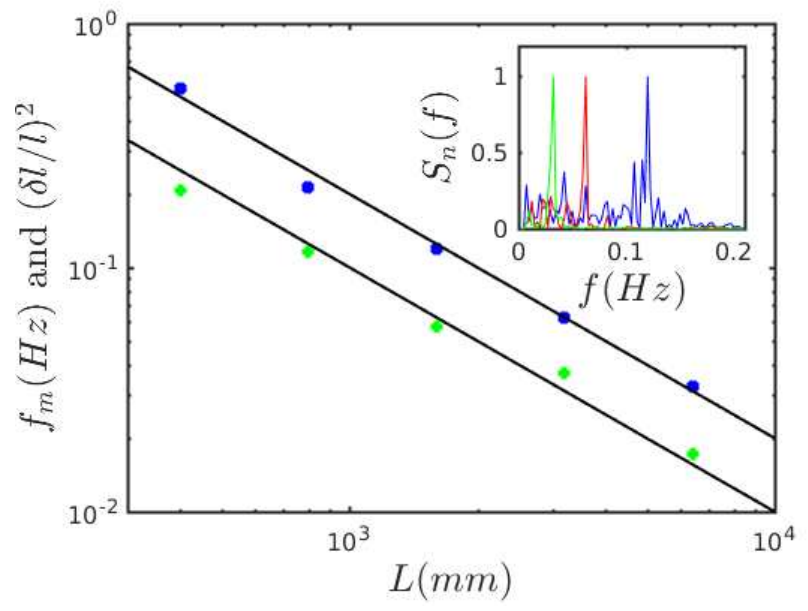

FIG. 4. The main panel shows the variation of frequency, $f$, (blue) and domain size fluctuations, $(\delta l / l)^{2}$, (green, scaled vertically for clarity) as a function of system length, $L$. The data shows that $f \sim L^{-1}$ and that $(\delta l / L)^{2} \sim L^{-1}$. The right inset shows the normalise power spectra, $S_{n}(f)$, as a function of frequency for three different length systems $(L=$ $1600 d, 3200 d$ and $6400 d$ ). There is a dominant frequency that scales as $L^{-1}$, as indicated in the main panel.

$l$, and Fourier transformed to get the frequency spectrum of the oscillations, shown in the inset to Fig. 4. We find that the frequency spectrum has a dominant frequency component, unlike the corresponding capillarywave spectrum observed in systems in thermodynamic equilibrium where the equipartition theorem is applicable [24]. The main plot shows this dominant frequency, $f_{m}$ (blue), as a function of the length $L$, for systems with the same mean density. The data shows that $f_{m}$ varies as $L^{-1}$, suggesting that wave-like sound propagation occurs [26]. The speed of sound is of order $0.2 \mathrm{~m} \mathrm{~s}^{-1}$, which is comparable with the typical velocity scale set by the vibrating cell, $A \omega=0.36 \mathrm{~m} \mathrm{~s}^{-1}$.

The green data in Fig. 4 shows that the relative amplitude of these oscillations $\delta l / l$ dies away as $\delta l / l \sim L^{-1 / 2}$. The fact that both $\delta l / l$ and $f_{m}$ tend to zero as $L \rightarrow \infty$ is our final piece of evidence indicating that the phase separated state is stable in the thermodynamic limit, in contrast to the corresponding equilibrium system which would break up into domains.

Our analysis shows that the granular gas can be described in terms of a reduced form of thermodynamics based on mechanical variables alone. The system evolves so as to minimise any residual mechanical work that can be released through inelastic collisions. Note that in thermodynamic equilibrium the principle of minimising mechanical work still holds. However, as energy is conserved the Maxwell construction must be evaluated along an isotherm. In this case minimising mechanical work is entirely equivalent to maximising entropy.

In the granular gas there is a decoupling of the kinetic and mechanical variables such that the appropriate 
equation of state, $p(v)$, is not an 'isotherm'. The minimisation of residual mechanical work allows a 1D dissipative gas to exhibit complex collective behaviour and emergent phenomena of the kind that are typical of far- from-equilibrium systems in general. As such it would be interesting to investigate whether our findings apply to other driven particle systems.
[1] H. Jaeger and A.J. Liu. arXiv:1009.4874v1 cond-mat.soft (2010).

[2] P Ball. The self-made tapestry: pattern formation in nature (Oxford University Press, 2001).

[3] I.S. Aranson and L.S. Tsimring, Rev. Mod. Phys., 78, 641 (2006).

[4] L. D. Landau and E. M. Lifshitz, Statistical Physics (Elsevier, 2013).

[5] For a review, see H.M. Jaeger, S.R. Nagel and R.P. Behringer. Rev. Mod. Phys., 68, 1259 (1996).

6] N. V. Brilliantov and T Poschel, Kinetic Theory of Granular gases (Oxford University Press, 2004).

[7] K. Röller, J.P.D. Clewett, R.M. Bowley, S. Herminghaus and M.R. Swift, Phys. Rev. Lett., 107, 048002 (2011).

[8] J.P.D. Clewett, K. Röller, R.M. Bowley, S. Herminghaus and M.R. Swift, Phys. Rev. Lett., 109, 228002 (2012).

[9] J.P.D. Clewett, J. Wade, R.M. Bowley, S. Herminghaus, M.R. Swift and M. G. Mazza, Scientific Reports 6, 28726 (2016).

[10] S. Herminghaus and M. G. Mazza, Soft Matter, 13, 898 (2017).

[11] A. P. Solon, J. Stenhammar, M. E. Cates, Y. Kafri, and J. Tailleur Phys. Rev. E 97, 020602(R) (2018).

[12] M. Argentina, M. G. Clerc and R. Soto, Phys. Rev. Lett.,
89, $044301(2002)$

[13] C. Cartes, M. G. Clerc and R. Soto, Phys. Rev. E, 70, 031302 (2004).

[14] R. Soto, M. Argentina, and M.G. Clerc, in Granular Gas Dynamics, edited by T. Pschel and N. Brilliantov (Springer, Berlin, 2003).

[15] E. Khain and B Meerson, Phys. Rev. E, 66, 021306 (2002).

[16] E. Khain, B. Meerson and P. V. Sasorov, Phys. Rev. E, 70, 051310 (2004)

[17] If the height of the cell is reduced the binodal/spinodal balloons shrink, similar to the behaviour discussed in [16].

[18] Finite Size Scaling and Numerical Simulation of Statistical Systems edited by V. Privman (World Scientific, 1989).

[19] For a movie see Supplementary Materials.

[20] A.J. Bray, Adv. Phys. 43, 357, (1994).

[21] M. P. Allen and D. J. Tildesley, Computer Simulation of Liquids (Oxford University Press, 2017).

[22] F. Mandl, Statistical Physics (Wiley \& Sons Ltd., 1985).

[23] J.-P. Hansen and L. Verlet, Phys. Rev., 184, 151 (1969).

[24] J.S. Rowlinson and B. Widom, Molecular theory of capillarity (Oxford University Press, 1982).

[25] H. Touchette, Physics Reports, 478, 1 (2009).

[26] M.G. Clerc et al. Nature Phys., 4, 249-254, (2008). 